\title{
The Effect of Supportive Educational Intervention on Perceived Stress and Severity of Chemotherapy-Related Neuropathy in Breast Cancer
}

\section{Patients}

\author{
Neda Arbabi ${ }^{1}$, Mohammad Kazem Momeni ${ }^{2}$, Pegah Sasanpour ${ }^{3}$ and Fatemeh Kiani ${ }^{4,}$ \\ ${ }^{1}$ Zahedan University of Medical Sciences, Zahedan, Iran \\ ${ }^{2}$ Department of Internal Medicine, Clinical Immunology Research Center, Ali Ibn Abitaleb Hospital, School of Medicine, Zahedan University of Medical Sciences, Zahedan, \\ Iran \\ ${ }^{3}$ Department of Surgery, School of Medicine, Zahedan University of Medical Sciences, Zahedan, Iran \\ ${ }^{4}$ Community Nursing Research Center, Zahedan University of Medical Sciences, Zahedan, Iran \\ "Corresponding author: Community Nursing Research Center, Zahedan University of Medical Sciences, Zahedan, Iran. Email: fkiani2011@yahoo.com
}

Received 2022 January 05; Accepted 2022 January 05.

\begin{abstract}
Background: The nature of breast cancer (BC) and its treatment is such that it leads to physical and psychological complications. Objectives: The present study aimed to determine the effect of supportive educational intervention on the perceived stress and severity of chemotherapy-related neuropathy in BC patients.

Methods: This quasi-experimental study was carried out on 60 women suffering from BC in Zahedan, Iran, in 2020. The patients were selected using the convenience sampling method and randomly assigned into two groups of intervention and control. The patients in the intervention group received four 45-minute sessions about the common problems of BC on a weekly and individual basis. The control group received only routine ward care. Data collection tools were the Perceived Stress Scale (PSS) developed by Cohen and Leeds Assessment of Neuropathic Symptoms and Signs (LANSS) Pain Scale. Data were collected before the intervention and six weeks after the intervention. Data analysis was performed using SPSS 22 software.

Results: The results of independent $t$-test showed that the mean and standard deviation of perceived stress score in the intervention group decreased from $56.8 \pm 5.92$ to $50.36 \pm 3.89(\mathrm{P}<0.001)$, and in the control group decreased from $55.6 \pm 3.65$ to $54.8 \pm 3.53(\mathrm{P}=$ 0.258). The mean and standard deviation of neuropathy severity score in the intervention group decreased from $12.90 \pm 1.66$ to 8.43 $\pm 2.16(\mathrm{P}<0.001)$, and in the control group increased from $12.56 \pm 2.28$ to $13.03 \pm 1.93(\mathrm{P}=0.276)$. The independent $t$-test showed that after implementing the supportive educational intervention, there was a significant difference between the two groups in terms of mean and standard deviation of neuropathy severity score $(\mathrm{P}<0.001)$.

Conclusions: According to our results, supportive educational intervention reduced the perceived stress and severity of neuropathy. Thus, it is necessary to include these supportive interventions in educational and care programs to improve patients' psychological status and reduce stress and neuropathy.
\end{abstract}

Keywords: Education, Support, Stress, Neuropathy, Breast Cancer

\section{Background}

Cancer is a disease process in which cells proliferate abnormally and ignore growth-regulating signals around the cells (1). The disease affects people of all ages, cultures, races, and socioeconomic classes. Some of the cancers have a significant impact on the quality of life of patients due to the disabilities they cause (2). In Iran, cancer is known as the third leading cause of death after cardiovascular diseases and accidents, accounting for more than 30,000 deaths annually (3). Breast cancer (BC) is the most common malignant disease of women worldwide and ac- counts for $28 \%$ of all cancers (4). Annually, more than 1.1 million new cases of $\mathrm{BC}$ are reported, accounting for about $10 \%$ of all new cancers and $23 \%$ of all women's cancers (5). The most common age of the onset of the disease in Iranian women is 47 years, which is one decade lower than the age in the developed countries. About $76 \%$ of the most common women's cancer cases in Iran are $\mathrm{BC}$, and the incidence of BC in Iranian women is 22 per 100,000 , and the prevalence is 120 per 100,000 people, which is a very shocking statistic. The total number of BC patients in Iran is 40,000 , and more than 7,000 patients are added to this number annually (6). 
One of the most important treatments of BC that kills $90 \%$ of malignant cells is chemotherapy, which is widely used to increase the survival rate with as little damage as possible (3). The use of chemotherapy drugs is generally associated with various serious and non-serious complications (7). One of the important complications of chemotherapy that is far from the view of the treatment staff is neuropathy. Neuropathic pain is a chronic pain that results from damage to the central or peripheral nerves. In this type of injury, the sensory and motor fibers, as well as the receiving field of the fibers, are disturbed, and its most important symptom is increase in pain (8), which is in the form of spontaneous burning pain at the site of injury, hyperalgesia (increased response to a stimulus that is normally painful) and allodynia (a response to a stimulus that does not cause pain normally) (9). Peripheral neuropathy is an important complication of chemotherapy that occurs following the use of drugs such as taxanes, which are mainly used in the treatment regimens of women with BC $(10,11)$. Neuropathy of these drugs manifests itself in the form of peripheral sensory neuropathy with symptoms such as distal paresthesia of the limbs, loss of sensation, and weakening of deep tendon reflexes (12). With the increasing number of women with BC, it is necessary to pay attention to the complications of neuropathic pain that have negative and adverse effects on various aspects of their lives. In many patients with neuropathy, pain is a common symptom. Symptoms of the disease are usually concentrated in the lower limbs and first in the soles of the feet and toes, which in addition to causing discomfort affects all aspects of their lives. It can be said that BC is associated with a lot of stress. On the other hand, the side effects of the treatment have a double effect on patients' perceived stress $(13,14)$. Stress refers to situations or events that a person faces in life but are not in harmony with the current facilities and capabilities of the person; therefore, she/he suffers from psychological conflict (15). Stress can increase tumor growth by affecting the immune defenses and the tumor itself; it can also lead to poor diet and sleep, as well as less physical activity. Finally, women who experience stress following BC are less likely to follow their doctor's recommendations, instructions, and prescriptions (16). In addition to medication, non-pharmacological measures, such as patient education, may help reduce the symptoms of neuropathy and stress to some extent. There are several methods used to educate patients, but one of the comprehensive educational programs is supportive educational package, which considers all the physical and mental dimensions of patients. In fact, supportive educational programs are a process in which individuals and patients learn to behave in a way that promotes, maintains, and improves health $(17,18)$. So, social support is the interaction between the provider and the recipient of support (19). In other words, social support includes emotional, instrumental, information, and evaluation support. While emotional support emphasizes the empathetic relationships with members of the social network, instrumental support refers to routine activities (20). Nurses, as the most important members of the treatment team, have an important role in caring for cancer patients under treatment and are in a good position to train and support them due to their long-term contact (21).

\section{Objectives}

The present study aimed to investigate the effect of supportive educational intervention on perceived stress and severity of chemotherapy-related neuropathy in patients with BC.

\section{Methods}

The present quasi-experimental study included two groups with a pre-test and post-test design. The study was approved by the ethics committee of Zahedan University of Medical Sciences (code: IR.zaums.REC.1398.516). The sample size was determined as 30 individuals in each group based on similar studies with $95 \%$ confidence interval, 95\% statistical test power, and considering the possible dropout (22).

$n=\frac{\left(Z_{1-\frac{\alpha}{2}}+Z_{1-\beta}\right)^{2} \times\left[S_{1}^{2}+S_{2}^{2}\right]^{2}}{\left(\bar{X}_{1}-\bar{X}_{2}\right)}$

$=30$

$$
Z_{1-\frac{\alpha}{2}}=1.96 ; \mathrm{S}_{1}=5.28 ; \bar{X}_{1}=7.67 ; Z_{1-\beta}=1.61 ; \mathrm{S}_{2}=3.49
$$

$; \bar{X}_{2}=16.90$

In this study, 60 cancer patients undergoing chemotherapy in the oncology wards of Khatam alAnbia and Ali Ibn Abitaleb (AS) hospitals in Zahedan, Iran, were selected. The patients were selected by convenience sampling method and randomly assigned into two groups of intervention and control.

The inclusion criteria were: definitive diagnosis of BC, being in the age range of 20 - 60 years, starting taxanes from the second session, having no metastatic malignancy, no addiction to drugs, having no mental illnesses and physical disability, not suffering from diabetes or grade 2 and 3 cancer, not being pregnant, being fluent in Persian, and having no other cancers. 
Data collection tools included a demographic information questionnaire, the Perceived Stress Scale (PSS) developed by Cohen, and the Leeds Assessment of Neuropathic Symptoms and Signs (LANSS) Pain Scale. The demographic information questionnaire included information such as age, occupation, level of education, duration of suffering from cancer, disease stage, and body mass index (BMI) (Table 1). The PSS consists of 14 items to measure general perceived stress in the past month. The questionnaire is scored on a 5-point Likert Scale with a minimum score of zero and a maximum score of 56. A higher score indicates more perceived stress. The reliability of this questionnaire was 0.80 (23) in the research by Sepahvand. In the current study, the questionnaire reliability was determined as 0.85 .

\begin{tabular}{|c|c|c|c|}
\hline Variable & Intervention & Control & P-Value \\
\hline Age & $48.3 \pm 10.36$ & $51.36 \pm 7.80$ & $0.145^{\mathrm{b}}$ \\
\hline Duration of illness $(y)$ & $1.94 \pm 2.47$ & $1.37 \pm 1.80$ & $0.130^{\mathrm{b}}$ \\
\hline Body mass index (BMI) & $21.16 \pm 3.68$ & $26.48 \pm 6.04$ & $0.597^{\mathrm{b}}$ \\
\hline Disease stage & & & $0.432^{c}$ \\
\hline Stage 2 & $16(53.3)$ & $19(63.3)$ & \\
\hline Stage 3 & $14(46.7)$ & $11(36.7)$ & \\
\hline Education level & & & $0.683^{c}$ \\
\hline High school diploma or less & $23(76.7)$ & $23(76.7)$ & \\
\hline $\begin{array}{l}\text { Diploma and above } \\
\text { diploma }\end{array}$ & $7(23.3)$ & $7(23.3)$ & \\
\hline Employment status & & & $0.399^{c}$ \\
\hline Employed & $4(13.3)$ & $2(6.6)$ & \\
\hline Unemployed & $26(86.7)$ & $28(93.4)$ & \\
\hline
\end{tabular}

The LANSS Pain Scale has seven items and assesses the severity of neuropathy. Scores range from 0 to 24 , and scores above 12 indicate a higher severity of neuropathy. The reliability of this questionnaire was obtained in Bennett's study with a Cronbach's alpha coefficient of 0.74 (24). In the current study, the questionnaire reliability was determined as 0.86 . Before the intervention, the LANSS and the PSS were completed by the patients in both groups. The questionnaires were completed through interviews. Patients in the control group only received the ward's routine care. In the intervention group, in addition to the routine care, from the second session of starting taxanes in the chemotherapy regimen, the researcher provided the patients with the necessary supportive educational measures based on the common problems of BC patients, including neuropathy, during four weekly sessions of 30 - 45 minutes in the ward. The educational measures were completed by question \& answer and face-to-face interviews using an educational booklet. In the sixth week, after the educational sessions, the questionnaires were completed again through interviews in the hospital. At the end of the study, the educational booklet was provided to the control group and the contact number of the researcher was provided to the control group. Research data was analyzed by SPSS Software (V22). First, frequency, percentage, mean, and standard deviation were determined using descriptive statistics. Shapiro-Wilk test was used to determine normality. The independent $t$-test was used to compare the means before and after the intervention between the two groups. The paired $t$-test was used to compare the means before and after the intervention in each group. The chi-square test was used to compare the frequency of qualitative variables between the two groups. The significance level in this study was less than 0.05 .

\section{Results}

Table 1 shows the demographic information of patients with $\mathrm{BC}$ in intervention and control groups.

Table 2 shows that the mean and standard deviation of perceived stress score decreased in the intervention group

The independent $t$-test showed that after implementing the supportive educational intervention, there was a significant difference between the two groups in terms of mean and standard deviation of stress score $(\mathrm{P}<0.001)$.

Table 3 shows that the mean and standard deviation of the neuropathic severity score decreased in the intervention group ( $\mathrm{P}<0.001)$, but increased in the control group $(\mathrm{P}=0.276)$. The independent $t$-test showed that after the implementation of supportive educational intervention, there was a significant difference between the two groups in terms of the mean and standard deviation of neuropathic severity score $(\mathrm{P}<0.001)$.

\section{Discussion}

The results of the present study showed that supportive educational intervention is effective in reducing the perceived stress and severity of neuropathy. Despite the importance of diagnosis and consequences of cancer, the existing policies in the hospital are limited to providing physical care, especially medication. Therefore, in the outpatient wards, in addition to physical care, paying attention to the psychological dimensions of the disease, including stress and drug side effects and its various aspects is of particular importance. In other words, the nonexistence of supportive educational interventions is very important. 
Arbabi Net al.

\begin{tabular}{lccc}
\hline Table 2. Comparison of Mean and Standard Deviation of Perceived Stress Score Before and After Implementation of Supportive Educational Intervention ${ }^{\text {a }}$ & Paired $t$-test \\
\hline Variable & Before Intervention & After Intervention & 0.001 \\
\hline Intervention & $56.8 \pm 5.92$ & $50.36 \pm 3.89$ & 0.258 \\
Control & $55.6 \pm 3.65$ & $54.8 \pm 3.53$ & 0.001 \\
Independent $t$-test & 0.349 & & \\
\hline
\end{tabular}

${ }^{\mathrm{a}}$ Values are expressed as mean $\pm \mathrm{SD}$. $(\mathrm{P}<0.001)$ more than of the control group $\left.(\mathrm{P}=0.258)\right)$.

Table 3. Comparison of Mean and Standard Deviation of Neuropathic Severity Score Before and After Implementation of Supportive Educational Intervention ${ }^{\mathrm{a}}$

\begin{tabular}{lcc}
\hline Variable & Before Intervention & After Intervention \\
\hline Intervention & $12.90 \pm 1.66$ & $8.43 \pm 2.16$ \\
Control & $12.56 \pm 2.28$ & $13.03 \pm 1.93$ \\
Independent $t$-test & 0.521 & 0.001 \\
\hline
\end{tabular}

${ }^{\text {a }}$ Values are expressed as mean $\pm \mathrm{SD}$.

The nature of cancer and its treatment is extremely stressful for patients. Studies show that the stress of these patients is related to the prognosis of treatment and unknown complications of the disease and the risk of involvement of other organs $(13,16)$. Therefore, performing supportive educational intervention in the ward can create communication and interaction between the patient and the treatment staff. On the other hand, raising the level of patient knowledge and performing supportive educational interventions by nurses can help reduce patients' perceived stress and concerns. In a study by Neisani Samani et al., supportive educational program was effective in reducing patients' stress (25). These results are consistent with those of the present study. Nevertheless, in their study the emphasis was mostly on information support, while in the present study, in addition to information support, practical and applied stress control methods such as relaxation, creative illustration, repetition of positive sentences, and breathing techniques were also taught. The study by Amir Zehani et al. revealed that although a considerable time has passed since the diagnosis and treatment of the disease, a significant number of patients suffer from the destructive psychological and emotional effects of diagnosis and treatment of their disease (26).

Due to the psychological effects associated with the diagnosis and treatment of cancer, the need for supportive educational interventions in different ways is felt more than ever. Besides, in a study by Emami et al., it was found that in addition to individual training, cognitivebehavioral stress management therapy had an effect on increasing resilience and stress control in women with BC (27). So, it can be said that the reason for the effectiveness of cognitive-behavioral intervention on increasing resilience is learning and acquiring skills, which help the patient to know how to face the inevitable problems and difficulties of life and have a better performance in coping with stressful situations (including cancer disease) and unpleasant events and enhancing skills in disease control and management (28). The results of a study by Ataollahi et al. on comparing dimension of perceived social support and perceived stress in women with and without BC showed that the perceived stress was higher in women with $\mathrm{BC}$ than in normal women $(\mathrm{P}=0.04)$, which is in a way consistent with the present study (29). The diagnosis and treatment of $\mathrm{BC}$ is associated with a lot of stress and anxiety. In addition, the cancer patients' exposure to stress increases the severity of the disease (30). Some studies have also emphasized that there is an inverse relationship between perceived stress and the pursuit of health behaviors in women with BC (31). Researchers believe that different intervention methods should be considered to identify and reduce the perceived stress symptoms in patients. This led to the use of different methods such as self-healing, which is one of the new approaches highly emphasizing stress control and management. Latifi et al. evaluated the effect of selfhealing training on stress, death anxiety, and depression in cancer patients and reported the effect of this educational method on controlling the perceived stress. In their study, self-healing was taught in 12 sessions, each of which lasted for 90 minutes. Their results showed the effect of this training method on controlling the perceived stress (32).

In addition to the mentioned methods, the results show the positive effect of group education of stress management methods in women with BC (33). The results of a study by Rezaei Ardani showed that cognitive-behavioral stress management training is effective in negative emotions and quality of life of women with BC (34). One of the most important issues affecting the perceived stress in 
these patients is the lack of control over the conditions and outcome of the disease. In general, the effect of the disease on the individual's role in society and life aggravates the psychological problems and disrupts the advancement of treatment programs. The general rule of supportive educational intervention is based on the principle that everyone could have a better understanding of their situation and, as a result, will feel more relaxed and in control, therefore those involved more actively in their self -management and prevention of disease recurrence.

In other words, using this type of intervention targets the feeling of control and belief in one's destiny as one of the important components. Therefore, the patients' participation in the treatment process and emphasis on creating a set of skills to manage perceived stress can improve the living conditions of patients and reduce stress. Overall, the results of the present study confirmed that supportive educational intervention can reduce perceived stress in patients with BC.

Due to the increase in the number of women with BC, it is necessary to pay attention to the complications of neuropathic pain, which has negative and adverse effects on various aspects of their lives, as neuropathic pain can reduce the patients' quality of life, cause frustration and unwillingness to continue treatment, and increase mental illnesses, stress, and sleep disorders (35). There is limited similar studies in the literature and most studies have been performed on other chronic diseases. In a study by Ahrary et al., the effect of supportive educational intervention on the symptoms and severity of mild to moderate diabetic peripheral neuropathy was examined in diabetic women. Although their study was slightly different from the present study in terms of methodology, the results were in line with our study (36). One of the important and influential factors affecting the severity of neuropathy is diet. Hence, one of the major concerns of the present study was educating patients in this field because studies show that the diets containing vitamin $\mathrm{D}$, which has been included in the educational content of the present study, can be somewhat helpful in controlling the patients' peripheral neuropathy. In this regard, Hosseini et al. showed the positive effect of vitamin D intake in controlling the symptoms associated with neuropathy (37). Consistent with the present study, Khanbabaei Gol et al. evaluated the prevalence of neuropathic pain and factors affecting sleep quality in women with BC after radiotherapy; however, the stage of intervention in the mentioned study was different from the present study. In their study, the intervention was performed during radiotherapy, while in the present study, the intervention was performed during chemotherapy. The results of their study also showed that neuropathy in women with BC leads to physical disorders (38). Usually, neuropathy is associated with chemotherapy and lasts for half of the life of patients, and radiotherapy has no role in exacerbating or intensifying it. However, since radiotherapy can also worsen the condition by causing certain side effects, it is necessary to pay special attention to this group of patients during the treatment period and even after it, because persistent neuropathy can severely affect the patient's living conditions (39). A study by Manas et al. examined patients 18 months after recovery and found that a history of chemotherapy caused neuropathic pain and psychological complications in patients (40). The abovementioned studies are in line with the present study. Studies show that progress in hyperalgesia (increased pain sensitivity) and decreased pain threshold lead to physical and psychological consequences (41). The results of studies emphasize that neuropathic pain can occur in patients with $\mathrm{BC}$ as a symptom of the disease, which is mainly due to the side effects of treatment, that is less addressed. Therefore, it is necessary to consider it. Apoptosis is one of the most common effects of medicines such as paclitaxel, which is caused by the cessation of cell mitosis (42). Peripheral polyneuropathy is one of the most common side effects of this class of medicines, that is caused by morphological or functional changes in peripheral nerves in the form of nerve axon disorders or myelin peripheral nerve disorders, but in fact the exact mechanism of this neuropathy has not been identified yet (43).

Clinically, medicines such as pregabalin and duloxetine are used to control neuropathic pains. In the present study, our patients received these medications for five weeks. However, despite the effect of pregabalin in controlling neuropathy, patients complain of some degree of neuropathy (44). It seems that since the pain caused by neuropathy occurs in patients with different spectrums, supportive educational interventions are needed to address the aspects and effects of this complication.

\subsection{Conclusions}

Due to the positive effect of supportive educational interventions on perceived stress and reducing the severity of neuropathy, it is necessary to include these interventions in educational and care programs, and the role of nurses in providing these services to patients is very important. Therefore, it is necessary for nurses to be available for providing information, and to strive for educating the client to improve their psychological status. It can also help to manage and control the psychological reactions of patients by attracting their cooperation and follow-up treatment, ultimately providing conditions for the control and relief of complications related to chemotherapy, especially neuropathy. 


\subsection{Limitations of the Study}

One of the most important limitations of this study was the time limit. Also, since this study was performed during the course of chemotherapy, generalizing the results to other therapeutic interventions such as radiotherapy and immunotherapy should be done with caution.

\subsection{Suggestions}

According to our results, neuropathy is one of the most important and lasting complications in BC patients. It is suggested that in future studies, these interventions be continued during the course of radiotherapy and even after discharge to determine the effect of interventions over time.

\section{Acknowledgments}

This article was extracted from an MSc thesis on Internal Surgery Nursing at the School of Nursing and Midwifery of Zahedan University of Medical Sciences, Iran. The authors wish to thank the Research Council of Zahedan University of Medical Sciences, the director of Ali Ibn Abitaleb and Khatam Al-Anbia Hospital, the head nurse, nurses, staff, and patients of the oncology ward of Khatam al-Anbia Hospital, and the chemotherapy ward of Ali Ibn Abitaleb Hospital.

\section{Footnotes}

Conflict of Interests: The authors declare that there is no conflict of interest.

Ethical Approval: The present quasi-experimental study included two groups with a pre-test and post-test design. The study was approved by the ethics committee of Zahedan University of Medical Sciences (code: IR.zaums.REC.1398.516).

Funding/Support: This study was funded by the ViceChancellor for Research of Zahedan University of Medical Sciences.

\section{References}

1. Hinkle JL, Cheever KH. Brunner and Suddarth's textbook of medicalsurgical nursing. Wolters Kluwer India Pvt Ltd; 2018.

2. Burns N, Grove SK. The practice of nursing research: Conduct. Critique. 2005.

3. Hasanvand S, Ashktorab T, Jafari Z, Salmani N, Safariyan Z. Cancerrelated fatigue and its association with health-related quality of. $A d v$ Nurs Midw. 2015;24(85):21-30.

4. KalanFarmanFarma K, Zareban I, Jalili Z, Shahrakipour M, Lotfi B. The effect of education on condition of knowledge, attitude and preventive behaviors of breast cancer in female teachers at guidance schools in Zahedan. J Torbat Heydariyeh Univ Med Sci. 2013;1(3):65-73.
5. Wolman I. Berek and Novak's Gynecology. 15th ed. Lippincott Williams and Wilkins; 2012.1560 p. doi: 10.1007/s13224-014-0538-z.

6. Etemadi A, Sajadi AR, Semnani SH, NOURAEI SM, Khademi H, Bahadori M. Cancer registry in Iran: a brief overview. Arch Iran Med. 2008.

7. Antony A, Joel JJ, Shetty J, Umar NF. Identification and analysis of adverse drug reactions associated with cancer chemotherapy in hospitalized patients. Int J Pharm Pharm Sci. 2016;8(7):448-51.

8. Dworkin RH, O'Connor AB, Backonja M, Farrar JT, Finnerup NB, Jensen TS, et al. Pharmacologic management of neuropathic pain: evidence-based recommendations. Pain. 2007;132(3):237-51. doi: 10.1016/j.pain.2007.08.033. [PubMed: 17920770].

9. Harden RN. Chronic neuropathic pain. Mechanisms, diagnosis, and treatment. Neurologist. 2005;11(2):111-22. doi: 10.1097/01.nrl.0000155180.60057.8e. [PubMed: 15733333].

10. Devita TH, Reserberg SAPA.Cancer Oprinciple and practice of Oncology.1. 7th ed. Philadelphia, USA: Lippincot, Williams and Wilkins; 2005. 995 p.

11. Windebank AJ. Chemotherapeutic neuropathy. Curr Opin Neurol. 1995;12(5):565-71. doi: 10.1097/00019052-199910000-00010. [PubMed: 10590893].

12. Lipton RB, Apfel SC, Dutcher JP, Rosenberg R, Kaplan J, Berger A, et al. Taxol produces a predominantly sensory neuropathy. Neurology. 1989;39(3):368. doi: 10.1212/wnl.39.3.368. [PubMed: 2564647].

13. Fontes F, Goncalves M, Pereira S, Lunet N. Neuropathic pain after breast cancer treatment and its impact on sleep quality one year after cancer diagnosis. Breast. 2017;33:125-31. doi: 10.1016/j.breast.2017.03.013. [PubMed: 28384563].

14. Golan-Vered Y, Pud D. Chemotherapy-induced neuropathic pain and its relation to cluster symptoms in breast cancer patients treated with paclitaxel. Pain Pract. 2013;13(1):46-52. doi: 10.1111/j.15332500.2012.00554.x. [PubMed: 22533683].

15. Bahadori Khosroshahi J, Khanjani Z. Relationship of humor and negative life events with depression among students. Zahedan J Res Med Sci. 2012;14(2).

16. Hernandez-Reif M, Field T, Ironson G, Beutler J, Vera Y, Hurley J, et al. Natural killer cells and lymphocytes increase in women with breast cancer following massage therapy. Int J Neurosci. 2005;115(4):495-510. doi: 10.1080/00207450590523080. [PubMed: 15809216].

17. Farahandi H, Mohebbi B, Tol A, Sadeghi R, Nori Jaliani K. The impacts of blended educational intervention program on blood pressure control and promoting HbAic among type 2 diabetic patients with hypertension. Razi J Med Sci. 2015;22(131):88-96.

18. Afshari M. The effect of BASNEF-based blended educational program on on diabetes control among type 2 diabetic patients referred to diabetes clinic of Samirom city. Razi J Med Sci. 2015.

19. Kang SW, Yoo JS. Health-promoting lifestyle and depression in metabolic syndrome patients in Korea. Int J Nurs Pract. 2012;18(3):26874. doi: 10.1111/j.1440-172X.2012.02036.x. [PubMed: 22621297].

20. Alizadeh Z, Ashktorab T, Nikravan Mofrad M, Zayeri F. Correlation between perceived social support and self-care behaviors among patients with heart failure. J Health Promot Manag. 2014;3(1).

21. Karbaschi K, Zareiyan A, Dadghari F, Siyadati SA. The Effect of SelfCare Program based on Orem's Theory on quality of Life of Cancer Patients Undergoing Chemotherapy in Military Personnel. Mil Caring Sci. 2015;2(2):69-77. doi: 10.18869/acadpub.mcs.2.2.69.

22. Khazaei T, Rezaei Mohabbati H, Sebzari AR, Hosseini M. The effect of foot reflexology massage on the stress and neuropathic pain caused by chemotherapy in women with breast cancer and genitalia.JBirjand Univ Med Sci. 2019;26(1):4-12.

23. Sepahvand T, Gilani B, ZAMANI REZA. The relationship between explanatory style.J Educ Psychol. 2009.

24. Bennett M. The LANSS Pain Scale: the Leeds assessment of neuropathic symptoms and signs. Pain. 2001;92(1):147-57. doi: 10.1016/s0304-3959(00)00482-6. 
25. Neisani Samani L, Hoseini AF, Modares Gilani M, Niroomand S. Effect of a Supportive Educational Program on Stress in Women with Endometrial Cancer. Iran J Nurs. 2018;30(110):11-20. doi: 10.29252/ijn.30.110.11.

26. Amir Zehani J.Quality of life and its relationship with perceived stress and self-confidence in women undergoing breast cancer treatment in Tabriz hospitals: descriptive-analytical cross-sectional study. Tabriz University of Medical Sciences; 1995.

27. Emami M, Askarizade G, Fazilat Pour M. Effectiveness of Cognitive: Behavioral Stress Management Group Therapy on Resilience And Hope in Women With Breast Cancer. J Posit Psychol Res. 2018;4(12):1-13.

28. Hasanzadeh Pashang S, Zare H, Alipor A. The efficacy of stress inculation training (SIT) on resilience, anxiety, depression and stress among spinal cord injury (SCI) patients. Pars Jahrom Univ Med Sci. 2012;10(3):15-26. doi:10.29252/jmj.10.3.15.

29. Ataollahi M, Masoumi SZ, Shayan A, Roshanaei G, Sedighi S. Comparing dimension of perceived social support and perceived stress in women with and without breast cancer referred to Mahdieh MRI Center of Hamedan in 2013. Pajouhan Sci J. 2016;14(2):62-70.

30. Folkman S, Lazarus RS, Dunkel-Schetter C, DeLongis A, Gruen RJ. Dynamics of a stressful encounter: Cognitive appraisal, coping, and encounter outcomes. J Pers Soc Psychol. 1986;50(5):992-1003. doi: 10.1037//0022-3514.50.5.992. [PubMed: 3712234].

31. Williams L, O'Connor RC, Howard S, Hughes BM, Johnston DW, Hay JL, et al. Type-D personality mechanisms of effect: the role of healthrelated behavior and social support.J Psychosom Res. 2008;64(1):63-9. doi:10.1016/j.jpsychores.2007.06.008. [PubMed:18158001].

32. Latifi Z, Sharifi Isfahani M. The Effect of self-Healing Training on Stress, Death Anxiety and Depression in Breast Cancer Patients. Iran J Psychiatr Nurs. 2021;9(3).

33. Mohammadi Arya A, Pakdaman M, Abolghasemi S, Rezaee M, Pashaee T, Ghareh Tapeh A. The effect of stress inoculation group training on hope and quality of life in women with breast cancer. J Res Dev Nurs Midw. 2014;11(1):8-16.

34. Rezaei Ardani A, Farid Hoseini F, Borhani Moghani M, Seyfi H. Cognitive behavioral stress management effects on quality of life and negative emotions in women with breast cancer. Iran J Obstet Gynecol Infertil. 2015;18(154).

35. Chehrehgosha M, Dastourpour M, Sanagu A, Mohamadi A. Cancer- related fatigue and its relationship with demographic and clinical characteristics. Jorjani Biomed J. 2013;1(2):24-31.

36. Ahrary Z, Khosravan S, Alami A, Najafi Nesheli M. The effects of a supportive-educational intervention on women with type 2 diabetes and diabetic peripheral neuropathy: a randomized controlled trial. Clin Rehabil. 2020;34(6):794-802. doi:10.1177/0269215520914067. [PubMed: 32306762].

37. Hosseini S, Asghari G, Fallah M, Yarjanli M, Hasibi Taheri S. Assessment of vitamin D efficacy on treatment of symptoms of patients with peripheral diabetic neuropathy admitted to Amiralmomenin Hospital in Tehran in 2015. Med Sci J Islamic Azad Univ Tehran Med Branch. 2017;27(1):24-31.

38. Khanbabaei Gol M, Rezvani F, Ghavami Z, Mobaraki-Asl N. Prevalence of neuropathic pain and factors affecting sleep quality in women with breast cancer after radiotherapy. Iran J Obstet Gynecol Infertil. 2019;22(6):46-53.

39. Jan M. New targets in pain, non-neuronal cells, and the role of palmitoyltalomide. Open Pain J. 2012;5(12). doi: 10.2174/1876386301205010012.

40. Manas A, Monroy JL, Ramos AA, Cano C, Lopez-Gomez V, Masramon $X$, et al. Prevalence of neuropathic pain in radiotherapy oncology units. Int J Radiat Oncol Biol Phys. 2011;81(2):511-20. doi: 10.1016/j.ijrobp.2010.05.047. [PubMed: 20832183].

41. Boakye PA, Olechowski C, Rashiq S, Verrier MJ, Kerr B, Witmans M, et al. A Critical Review of Neurobiological Factors Involved in the Interactions Between Chronic Pain, Depression, and Sleep Disruption. Clin J Pain. 2016;32(4):327-36. doi: 10.1097|AJP.0000000000000260. [PubMed: 26035521].

42. Yvon AM, Wadsworth P, Jordan MA. Taxol suppresses dynamics of individual microtubules in living human tumor cells. Mol Biol Cell. 1999;10(4):947-59. doi: 10.1091/mbc.10.4.947. [PubMed: 10198049]. [PubMed Central: PMC25218].

43. Barohn RJ. Approach to peripheral neuropathy and neuronopathy. Semin Neurol. 1998;18(1). doi: 10.1055/s-2008-1040857. [PubMed: 9562663].

44. Forogh B, Ebrahimi M, Mirshekari Z, Majidi L, Ghazai F, ESMAILI JM, et al. Comparing the effect of duloxetine with gabapentin in the treatment of diabetic peripheral neuropathy: A randomized clinical trial. Ebnesina. 2014. 